\title{
The Formation and Evolution of the Perseid Meteoroid Stream
}

\author{
Nathan W Harris ${ }^{1,2}$ \\ ${ }^{1}$ Astrophysics Research Group, School of Electrical Engineering, \\ Electronics \&3 Physics, Liverpool John Moores University, Byrom Street. \\ Liverpool, L3 3AF, UK.
}

\begin{abstract}
The orbital evolution of two modelled 'Perseid' meteoroid streams is investigated using direct numerical integration techniques. We conclude that, in the absence of significant meteoroid velocity determination errors, the observed meteoroid orbital semi-major axis distribution is a direct consequence of the cometary ejection process and not due to subsequent orbital evolution. A high ejection-velocity $\left(\sim 0.6 \mathrm{~km} \mathrm{~s}^{-1}\right)$ model stream succeeds in reproducing the observations. Conclusions are made concerning how the orbital stability of Earth-orbit-intersecting Perseid metecroids varies with initial orbital semi-major axis.
\end{abstract}

\section{Introduction}

A simple model has been developed to represent the formation of the Perseid meteoroid stream by considering the way in which both the velocity of the meteoroids emitted from the surface of the parent cometary nucleus (109P/SwiftTuttle) and the number ejected per unit time vary as a function of heliocentric distance and comet-Sun-meteoroid ejection angle (Harris et al. 1995). The IAU Meteor Data Centre at Lund (Lindblad 1991) contains a large data set of meteoroid orbits which allows us to gain reliable information on the distribution of the orbital elements for most of the major meteoroid streams. The $D^{\prime}$ criterion (Drummond 1981) can be used to select Perseid stream members from the stream candidates (e.g. Harris \& Hughes 1995). The orbital semi-major axis (a) distribution is shown in Figure 1 (i) (for a values up to $50 \mathrm{AU}$ ). There is considerable spread in the observed $a$ distribution, ranging from around $6 \mathrm{AU}$ to near parabolic orbits. The contrihution to the observed (measured) spread due to errors in the meteoroid velocity determination is unclear (the velocity errors maybe as high as $1 \%$ ) and is therefore ignored in the following work. This effect will be considered in a later paper. The distribution peak occurs between 12 and $18 \mathrm{AU}$ whereas the cometary orbital semi-major axis is around 26 AU The lower $a$ (and hence lower orbital period) meteoroids, however, intersect the Earth's orbit more frequently. An unbiased distribıtion could therefore be determined by multiplying the number of meteoroids in each histogram $a$ bin by the corresponding orbital period. Figure 1 (ii) shows a plot of meteoroid

\footnotetext{
${ }^{2}$ Present address : Armagh Observatory, College Hill, Armagh, Northern Ireland, BT61 9DG
} 
(i)

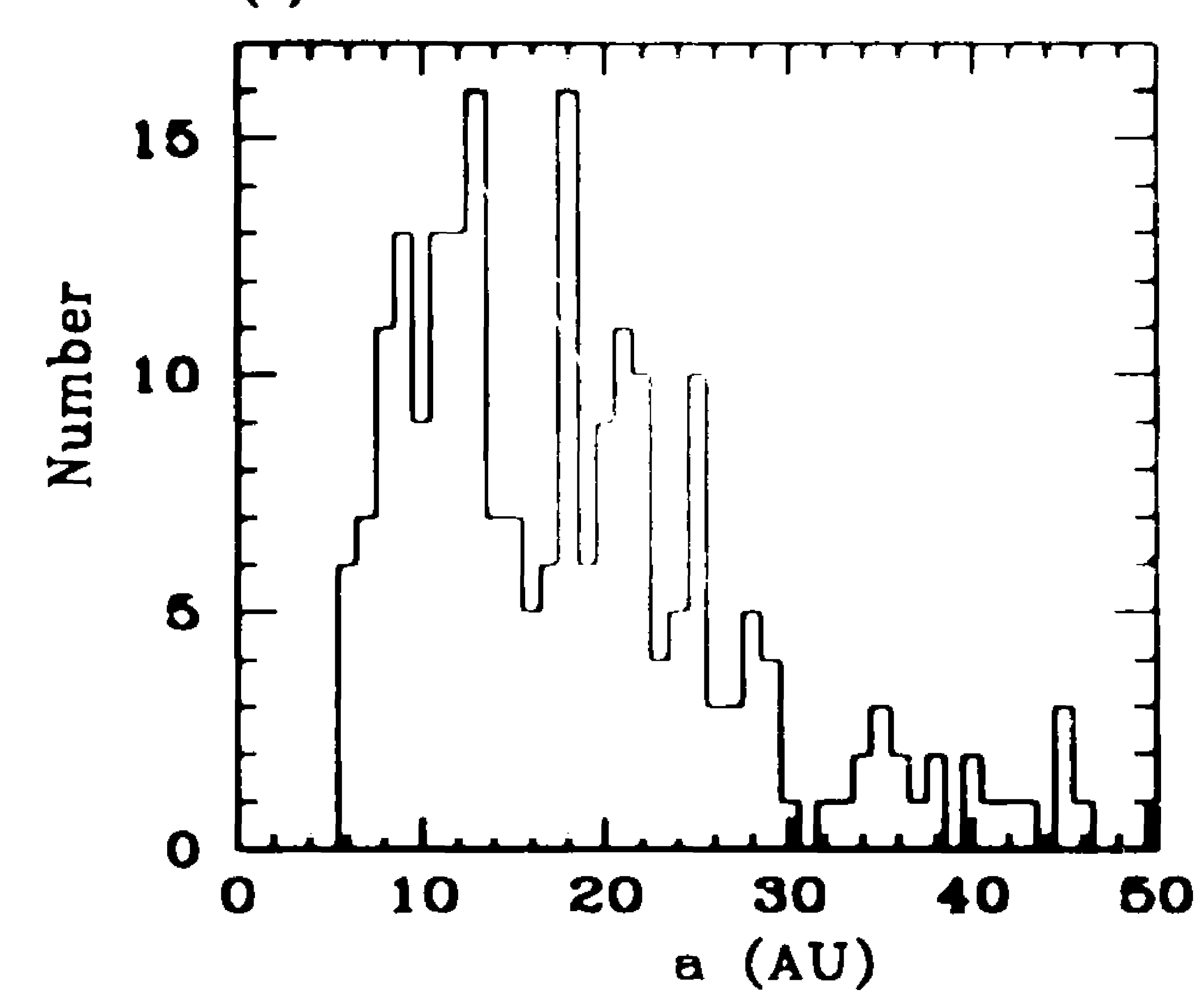

(ii)

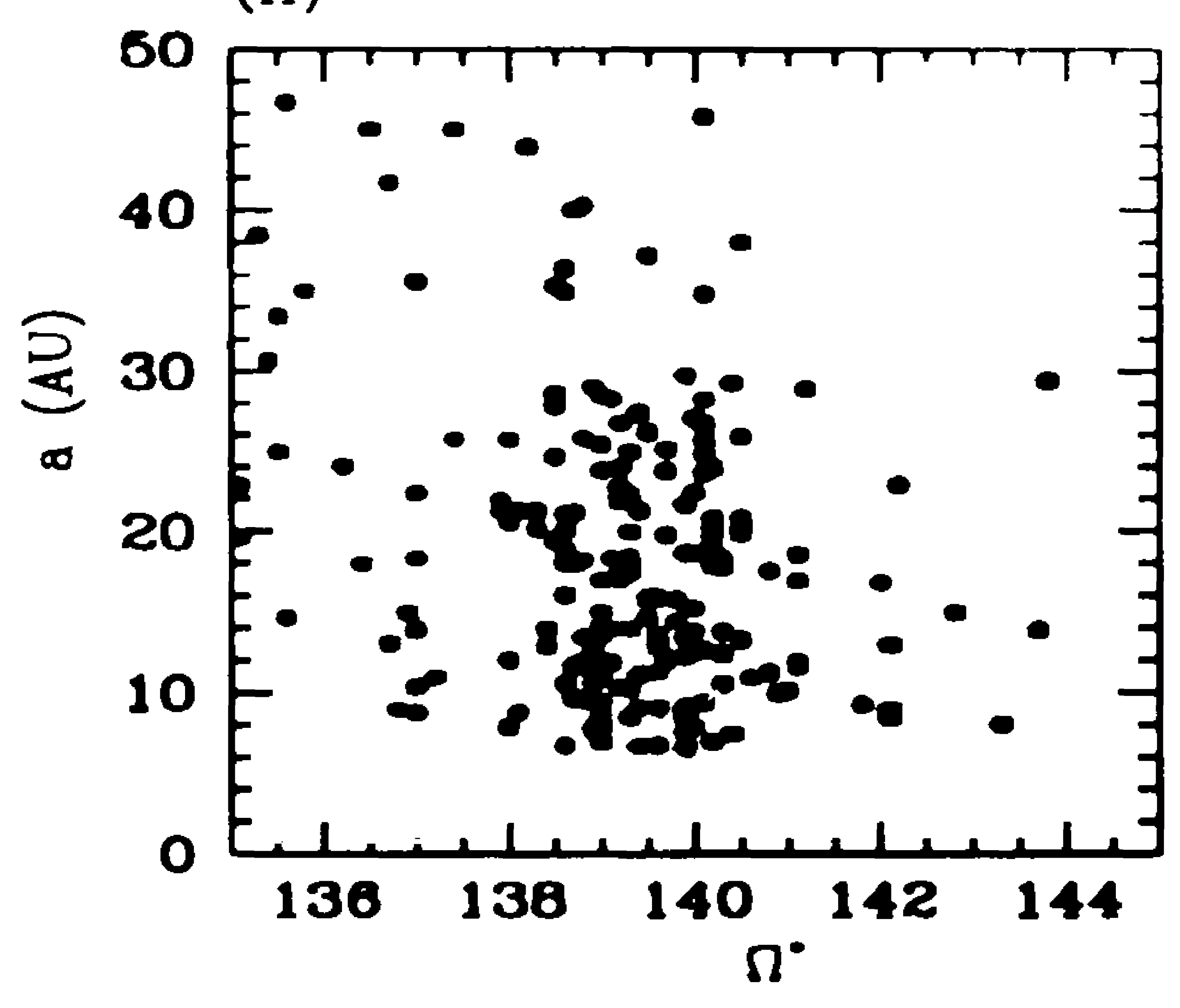

Figure 1. (i) The observed photographic Perseid meteoroid orbital semi-major axis distribution. (ii) A plot of $a$ versus the longitude of the ascending node for the observed Perseid meteoroids.

$a$ as a function of the longitude of the ascending node, $\Omega$. The figure clearly shows that there is no correlation between the two parameters indicating that those meteoroids that happen to intersect the Earth's orbit (and can potentially become visible as meteors) do not exhibit any special orbital semi-major axis values.

\section{Meteoroid Ejection and Orbital Evolution}

Whipple's formula for the ejection velocity of meteoroids from a cometary nucleus (Whipple 1951) results in an ejection velocity of order $0.05 \mathrm{~km} \mathrm{~s}^{-1}$ for meteoriods capable of producing 'typical' photographic Perseid meteors. (The 'typical' photographic Perseid meteor is caused by a meteoroid of mass $\sim 0.5 \mathrm{~g}$ and density around $0.3 \mathrm{~g} \mathrm{~cm}^{-3}$ ). In comparison the observed gas expansion velocities in cometary comae as a function of heliocentric distance is given by the relationship $V_{\text {gas }}=0.58 r^{-0.5}$, where $V_{\text {gas }}$ is in $\mathrm{km} \mathrm{s}^{-1}$ and $r$ is in AU (Delsemme 1982). At the perihelion distance of Swift-Tuttle, this results in a gas expansion velocity of around $0.6 \mathrm{~km} \mathrm{~s}^{-1}$. The solid-line histograms in Figure 2 show the resulting modelled meteoroid $a$ distributions produced by ejecting meteoroids at perihelion. in the plane of the parent comet's orbit (according to the model mentioned above), with a Maxwellian velocity distribution that peaks at a value of $0.05 \mathrm{~km} \mathrm{~s}^{-1}$. shown in Figure 2 (i) and $0.6 \mathrm{~km} \mathrm{~s}^{-1}$, shown in Figure 2 (ii). The cometary orbital parameters used were the mean values for the last 20 apparitions prior to 1862 (Yau et al. 1994). The high dust ejection velocities produce an $a$ distribution that resembles the observed distribution. The low dust ejection velocities produce an $a$ distribution with $20<a<38$ AU with a peak that coincides with that of the parent comet at around $26.58 \mathrm{AU}$. Comparing this with the observed distribution (Figure 1 (i)). it is clear that we require a mechanism for the systematic reduction of the modelled peak value to the observed peak value and also considerable spreading out of the distribution to higher and lower $a$ values.

The Poynting-Robertson effect is one such possible mechanism. The orbital lifetime of a 'typical' Perseid stream meteoroid (with initial values of $a$ and $e$ of $25 \mathrm{AU}$ and 0.969 respectively) under the PR effect (i.e. the time it takes the particle to spiral into the Sun) is approximately $2 \times 10^{9}$ years (Wyatt \& 
(i)

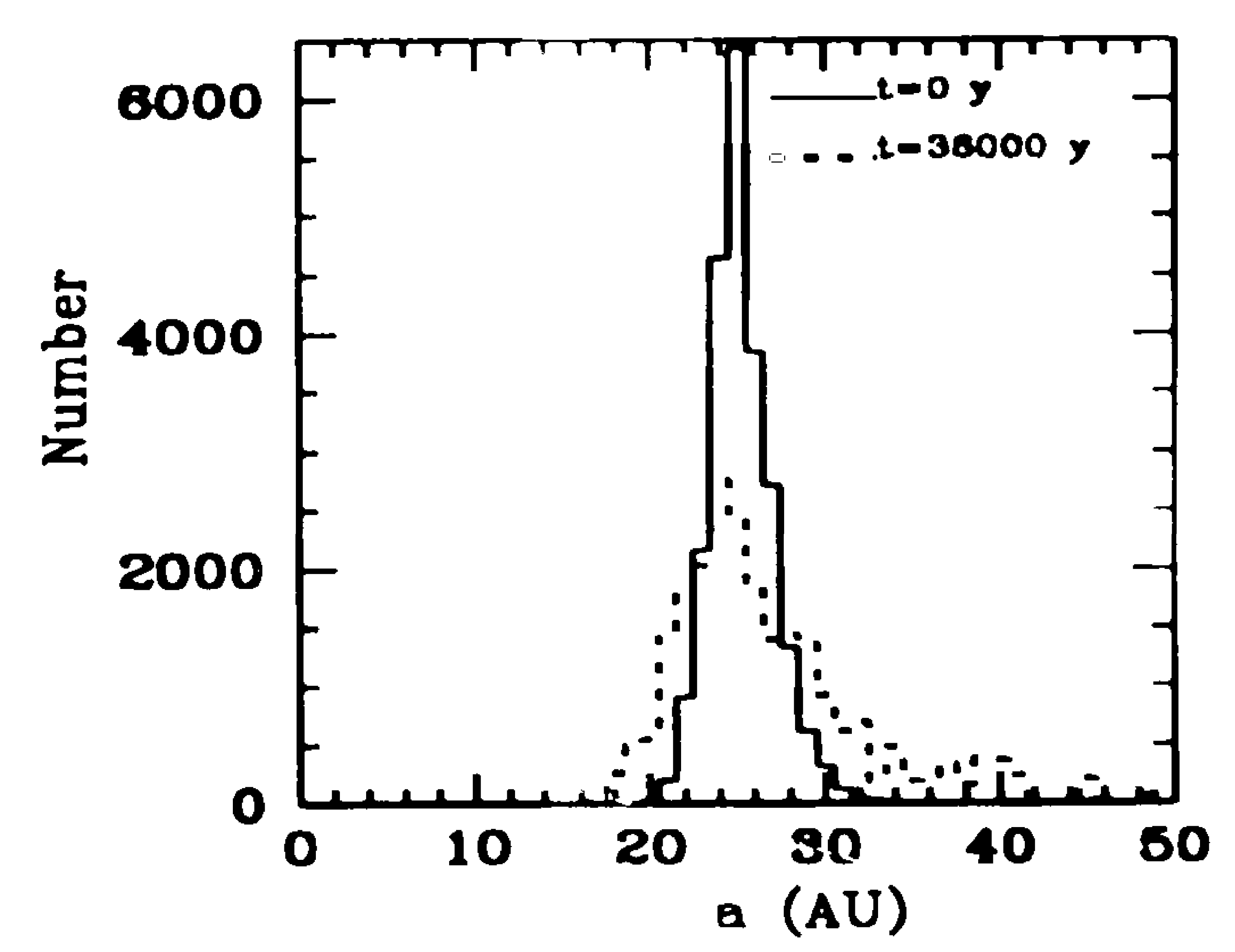

(ii)

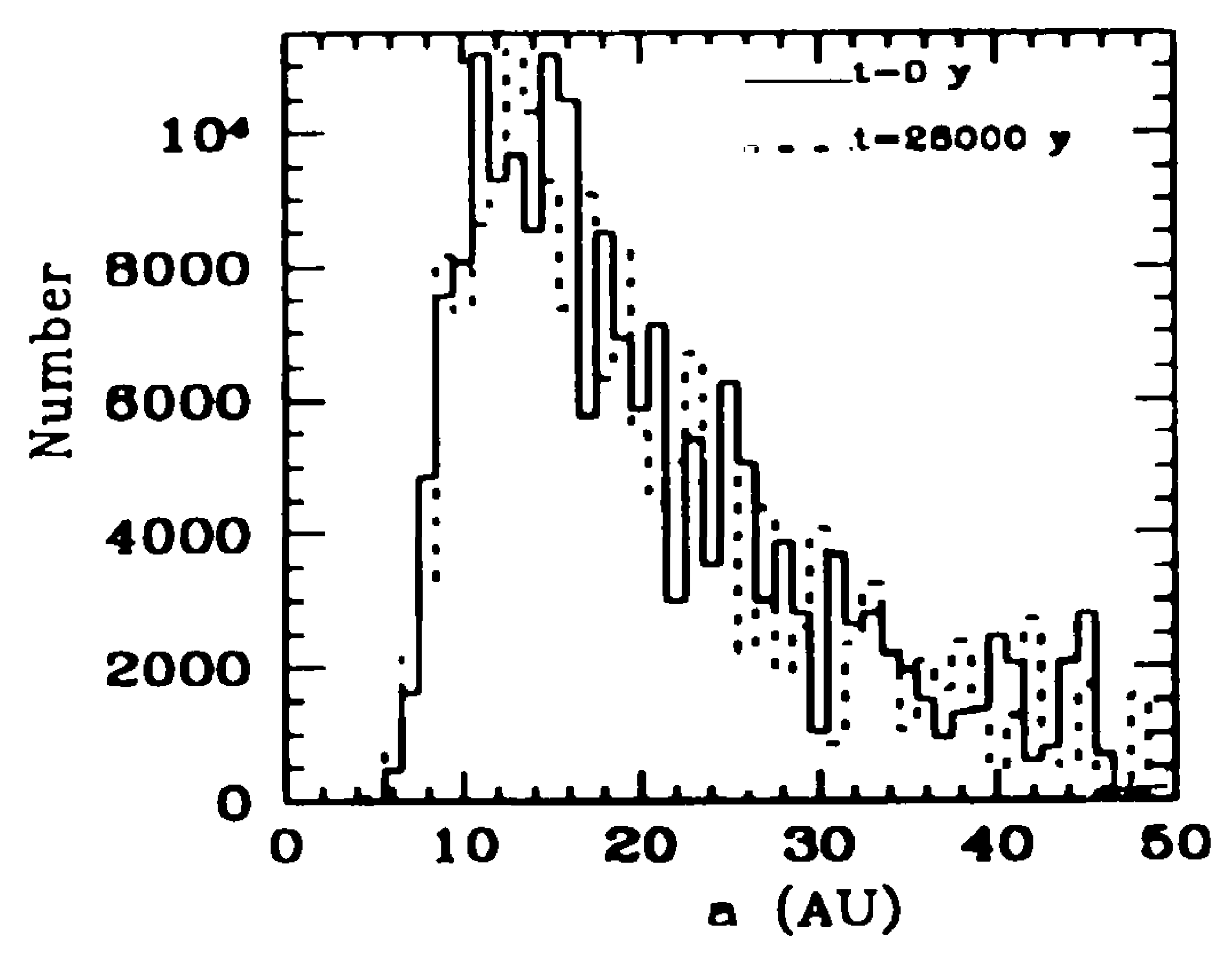

Figure 2. (i) Modelled meteoroid a distribution produced with mean ejection velocity of $0.05 \mathrm{~km} \mathrm{~s}^{-1}$ (solid-line histogram). The dottedline histogram shows the corresponding meteoroid $a$ distribution after 36000 years of gravitational perturbation. (ii) As for Figure 2 (i) but mean ejection velocity is $0.6 \mathrm{~km} \mathrm{~s}^{-1}$; perturbation time is $28000 \mathrm{y}$.

Whipple 1950). For an initial $a$ of $12 \mathrm{AU}(\mathrm{e}=0.92)$ the corresponding lifetime is around $5 \times 10^{8}$ years. As the collisional lifetime of a stream meteoroid is generally thought to be of the order $10^{5 \pm 1}$ years (Grün et al. 1984 , Steel \& Elford 1985) it is clear that the PR effect does not act swiftly enough to explain the discrepancy between the model and the observations.

Another possibility is that the presently observed meteoroids were ejected from a parent body with $a$ around 12 to $18 \mathrm{AU}$. Orbital integrations of SwiftTuttle, however, indicate that $a$ does not tend to decrease as we integrate backwards ir. time (Bailey \& Emel'yanenko 1995).

\section{Gravitational Perturbation and Conclusions}

A third mechanism is the systematic reduction of meteoroid $a$ due to gravitational perturbations. The modelled stream (containing $\sim 1000$ particles) was evolved for a few $\times 10^{4}$ years into the future using an orbital integration program based on the RADAU subroutine written by Everhart (1985). All planets from Earth to Neptune were taken into account and the masses of Mercury and Venus were added to that of the Sun. The Earth and Moon were treated as a single body. Figure 2 (i) (dotted-line histogram) shows the resulting meteoroid $a$ distribution after 36000 years of gravitational perturbation. It is evident that the overall effect is one of a gradual spreading out of the distribution with little movement in the distribution peak. This suggests that, in the absence of significant observational errors, the observed $a$ distribution is a direct consequence of meteoroid stream formation and not due to subsequent orbital evolution. This conclusion requires meteoroids to be ejected with high velocities, corresponding to model (ii). High ejection velocities could be obtained by massive meteoroids if the particles have non-spherical shapes and a high surface-area-to-mass ratio (Gustafson 1989). Figure 2 (ii) (dotted-line histogram) shows the meteoroid $a$ distribution for model (ii) after 28000 years of gravitational perturbation. The distribution as a whole is relatively stable over this time period, the only obvious movement being the 'sorting out' into various mean-motion resonances (mainly with Jupiter, eg. 1:n, where $n=3, \ldots 7$ ) (cf. Wu and Williams 1995). 


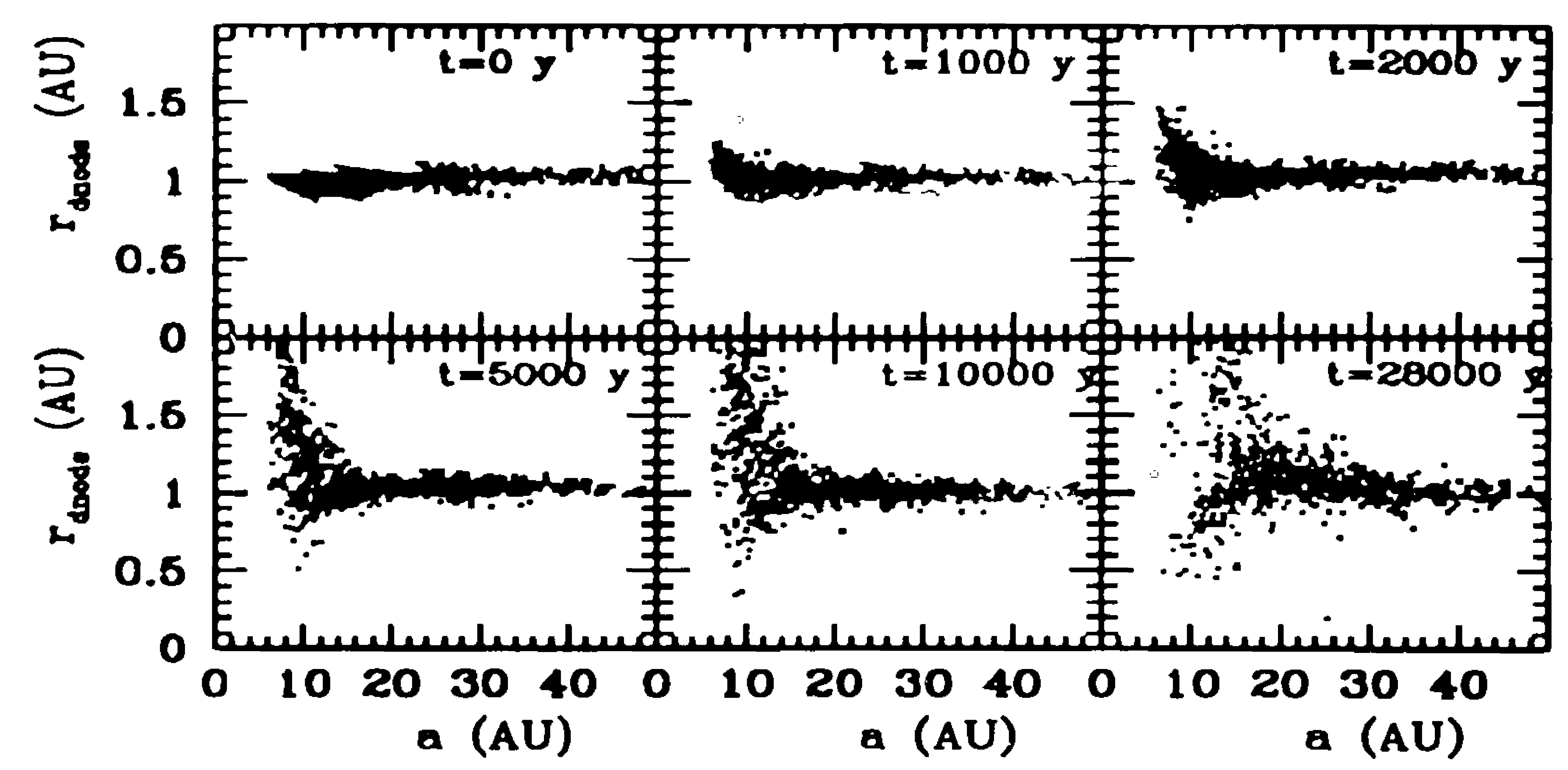

Figure 3. Plots of the heliocentric distance of the descending node $\left(r_{\text {dnode }}\right)$ against $a$ for specific epochs during the meteoroid stream evolution.

Examination of Figure 3, however, indicates a correlation between the stream dispersion rate and the initial meteoroid $a$. At time $t=0$ (i.e. soon after ejection) meteoroids with orbital descending nodes at the Earth's orbit, $r_{d n \text {.ode }} \simeq 1$ $\mathrm{AU}$, (i.e. those which can potentially be seen as meteors) are represented by the whole spectrum of $a$ values. As we follow the orbital evolution forwards it is clear that the lower $a$ meteoroids $\left(a<15 \mathrm{AU}\right.$ ) have their $r_{d n o d e}$ perturbed to heliocentric distances lying outside the Earth's orbit over a much shorter timescale than the higher $a$ meteoroids. This means that the observed low $a$ meteoroids are relatively young and must have been ejected from the cometary nucleus within the last few thousand years $(<50$ cometary orbital periods). The higher $a$ meteoroids can have much longer observable lifetimes ( $>10000 \mathrm{y}$ ).

\section{References}

Bailey, M.E. \& Emel'yanenko, V V. 1995, MNRAS, in press

Delsemme, A.H. 1982, in Comets, L.L. Wilkening, Univ of Arizona Press, 85

Drummond, J.D. 1981, Icarus, 45, 545

Everhart, E. 1985, in Dynamics of Comets: Their Origin and Evolution, A. Carusi \& G.B. Valsecchi, IAU Coll. No. 83, p. 185, Reidel

Grün, E., Zook, H.A., Fechtig, H. \& Giese, R.H. 1985, Icarus, 62, 244

Gustafson, B.A.S. 1989, ApJ, 337, 945

Harris, N W. \& Hughes, D.W 1995, MNRAS, 273, 992

Harris, N.W.. Yau, K.C. \& Hughes, D.W 1995, MNRAS, 273, 999

Lindblad, B.A. 1991, in Origin and Evolution of Interplanetary Dust, A.C.

Levasseur-Regourd \& H. Hasegawa. Kluwer Acad. Publishers, 311

Steel, D.I. \& Elford, W.G. 1985, MNRAS, 217

Whipple. F.L. 1951. ApJ, 113, 464

Wu, Z. \& Williams. I.P 1995, MNRAS, 276, 1017

Wyatt, S.P.. Jr \& Whipple, F.L. 1950, ApJ, 111. 134

Yau, K.C., Yeomans, D. \& Weissman, P 1994. MNRAS. 266, 305 